\title{
Energy Dissipation and the High-Strain Rate Dynamic Response of Vertically Aligned Carbon Nanotube Ensembles Grown on Silicon Wafer Substrate
}

\author{
P. Raju Mantena, ${ }^{1}$ Tezeswi Tadepalli, ${ }^{1}$ Brahmananda Pramanik, ${ }^{1}$ Veera M. Boddu, \\ Matthew W. Brenner, ${ }^{2}$ L. David Stephenson, ${ }^{2}$ and Ashok Kumar ${ }^{2}$ \\ ${ }^{1}$ Composite Structures and Nano-Engineering Research, Department of Mechanical Engineering, University of Mississippi, \\ University, MS 38677, USA \\ ${ }^{2}$ U.S. Army Engineer Research and Development Center-Construction Engineering Research Laboratory (ERDC-CERL), \\ Champaign, IL 61821, USA
}

Correspondence should be addressed to P. Raju Mantena; meprm@olemiss.edu

Received 14 March 2013; Revised 3 August 2013; Accepted 12 August 2013

Academic Editor: Teng Li

Copyright (c) 2013 P. Raju Mantena et al. This is an open access article distributed under the Creative Commons Attribution License, which permits unrestricted use, distribution, and reproduction in any medium, provided the original work is properly cited.

\begin{abstract}
The dynamic mechanical behavior and high-strain rate response characteristics of a functionally graded material (FGM) system consisting of vertically aligned carbon nanotube ensembles grown on silicon wafer substrate (VACNT-Si) are presented. Flexural rigidity (storage modulus) and loss factor (damping) were measured with a dynamic mechanical analyzer in an oscillatory threepoint bending mode. It was found that the functionally graded VACNT-Si exhibited significantly higher damping without sacrificing flexural rigidity. A Split-Hopkinson pressure bar (SHPB) was used for determining the system response under high-strain rate compressive loading. Combination of a soft and flexible VACNT forest layer over the hard silicon substrate presented novel challenges for SHPB testing. It was observed that VACNT-Si specimens showed a large increase in the specific energy absorption over a pure Si wafer.
\end{abstract}

\section{Introduction}

Functionally graded materials (FGMs) are a new generation of engineered materials wherein the microstructural details are spatially varied through nonuniform distribution of the reinforcement phase(s). These FGMs are being investigated for applications in blast, ballistic protection of building structures and other armor applications. A fundamental knowledge and understanding of the dynamic behavior of vertically aligned carbon nanotubes (VACNTs) with functionally graded stiffness modulus is needed to understand the energy transfer mechanisms and their dependence on the microstructures of carbon nanotube (CNT) ensembles. This information is expected to lead to novel, strong, lightweight, and robust composites for enhanced protection.

An in-depth study of the dynamic behavior of FGMs is accomplished by using reinforcements with different properties, sizes, and shapes, as well as by interchanging the roles of the reinforcement and matrix phases in a continuous manner. The result is a microstructure that produces continuously or discretely changing thermal and mechanical properties at the macroscopic or continuum scale. Thus FGMs are designed for optimal performance when functional requirements vary with location. This concept of engineering the material's microstructure allows designers to fully integrate the material and structural considerations into the final design of structural components.

The concepts of FGMs have also been extended to functionally graded ceramic-metal hybrids (ceramets), cementitious materials, and piezoelectric materials as well. Functionally graded materials are ideal candidates for applications involving severe thermal gradients, ranging from thermal structures in advanced aircraft and aerospace engines to computer circuit boards. High flexural rigidity with high 
vibration damping is a desirable property for aerospace and automotive applications to ensure a large load-bearing capacity and increased fatigue life.

Nanometer-scale structures such as carbon nanotubes (CNTs) and nanowires with exceptional thermal, electronic, and mechanical properties are undergoing intense experimental and theoretical investigations for a large range of possible applications. With the ready availability of freestanding VACNTs, integrating high flexural rigidity and damping into structures is feasible. Increasing the energy dissipation characteristics by introducing stiffness gradients through topological defects and entanglements of the CNTs will also be beneficial in designing shock and impact resistant light-weight protection systems. In this paper, the dynamic mechanical behavior and high-strain rate dynamic response characteristics of a functionally graded material system consisting of vertically aligned carbon nanotubes grown on a silicon wafer substrate (VACNT-Si) are presented.

\section{Materials and Methods}

2.1. VACNT-Si Specimen Preparation. A well-established chemical vapor deposition approach is used for the synthesis of the VACNTs on silicon wafers [1-4]. The carbon source, anhydrous $\mathrm{m}$-xylene (purity $\geq 99 \%$ ), and the catalyst, ferrocene (purity: 98\%) were obtained from Sigma Aldrich, St. Louis, MO, USA. Ultra-high-purity nitrogen gas and ultrahigh-purity $99.999 \%$ hydrogen were supplied by S.J. Smith Welding Supply, Urbana, IL, USA. Silicon wafers, $100 \mathrm{~mm}$ (3.94 inches) diameter type $\mathrm{P}$ boron doped, were purchased from Wafer World Inc., Palm Beach County, FL, USA. The silicon wafers were surface cleaned and etched with nitric acid (Fisher Scientific, Gardena, CA, USA) and hydrofluoric acid (Mallinckrodt Chemicals, Phillipsburg, NJ, USA). Reagent grade deionized water (Thermo Scientific, Middleton, VA, USA) was used for dilutions and rinsing and during silicon wafer dicing. Silicon wafers $6 \mathrm{~mm}$ ( 0.24 inches $) \times 55 \mathrm{~mm}(2.17$ inches) are prepared by first coating the wafer in photoresist to protect it from the dicing process. Then using a diamond saw, the wafers are diced into required sample sizes. To remove the photoresist, the samples are sonicated in acetone for 2 minutes and then transferred into a new acetone bath where they are sonicated for an additional 2 minutes.

The samples are cleaned by sonicating them in nitric acid for 2 minutes and then in deionized water for 2 minutes. Surface etching is done by agitating the samples in $51 \%$ hydrofluoric acid for $60 \mathrm{~s}$. Immediately the sample is extracted and rinsed in deionized water for 2 minutes, and then rinsed in another bath of deionized water for 2 minutes. The samples are dried using forced air. The preparation of the catalytic solution involves dissolving ferrocene in the $\mathrm{m}$-xylene solvent. The mixture is agitated and then finally sonicated to fully dissolve the ferrocene powder. The concentration of ferrocene is $5 \%$ by weight.

The cleaned and etched Si substrate is then placed in a quartz tube ( $2^{\prime \prime}$ diameter, $38^{\prime \prime}$ long). The tube is tilted at a $2^{\circ}$ angle to facilitate CNT growth on the Si surface and prevent clogging at the injection point. A bundle of glass wool is also inserted in the inlet portion of the tube to

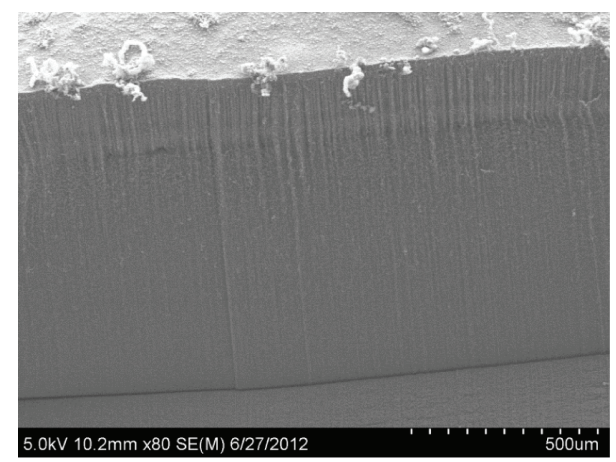

FIGURE 1: Scanning electron micrograph of vertically aligned carbon nanotubes (VACNTs) grown on a silicon wafer using the chemical vapor deposition approach.

create a more uniform flow profile. The tube is flushed with a $2500 \mathrm{~mL} / \mathrm{min}$ flow of nitrogen to remove oxygen. Under the flow of nitrogen, the tube is heated in a programmable tubular furnace (GSL-1500x, MTI, Richmond, VA, USA) from room temperature to $300^{\circ} \mathrm{C}$ in $30 \mathrm{~min}$ and then brought from 300 to the process temperature $820^{\circ} \mathrm{C}$. During this time, a heating tape is wrapped around the inlet portion of the quartz tube to preheat the gases and vaporize the carbon source and ferrocene solution and allowed to reach about $350^{\circ} \mathrm{C}$.

Once the processing temperature is reached, the temperature is fixed within $1^{\circ} \mathrm{C}$ and the furnace is held for a minimum of 15 minutes at the process temperature before CNT growth is initiated to ensure that the sample has been properly heated. The hydrogen gas is then turned on and tuned to a flow rate of $200 \mathrm{~mL} / \mathrm{min}$ and allowed to stabilize for approximately 5 minutes. The solution of $\mathrm{m}$-xylene and ferrocene is then injected at a constant flow rate of $\sim 16 \mathrm{~mL} / \mathrm{hr}$ which continues for $60 \mathrm{~min}$. After this period of processing, the flow of solution is terminated, the furnace heating power is shut off, the hydrogen gas is shut off, and the inlet heating power is turned off. Nitrogen is still flowed through the tube to aid in cooling and preventing oxygen and other contaminants from entering the tube. Once the tube has reached room temperature, the nitrogen flow is shut off and the CNT coated Si sample is removed for measurement. A typical scanning electron micrograph (SEM) of the synthesized VACNTs is shown in Figure 1.

2.2. Dynamic Mechanical Analysis. The dynamic mechanical properties $[5,6]$ of pure Si wafer and VACNT-Si were analyzed with a TA Instruments Model Q800 DMA. The pure silicon wafer rectangular beam specimen was $6 \mathrm{~mm}(0.24$ inches) in width, $0.4 \mathrm{~mm}$ (0.016 inches) in thickness and $55 \mathrm{~mm}$ (2.17 inches) in length. VACNT forest was grown on one side of the pure silicon wafer beam to a height of about $0.5 \mathrm{~mm}$ (0.02 inches) (Figures 2(a) and 2(b) inset). A threepoint oscillatory bending mode was utilized for evaluating the dynamic modulus and loss factor (damping) of both the pure Si wafer and the VACNT-Si specimens.

The specimens were simply supported at the ends on two roller pins (Figure 2(a)) with a span of $50 \mathrm{~mm}$ (1.97 inches) and loaded from top with a fixed roller pin positioned at 

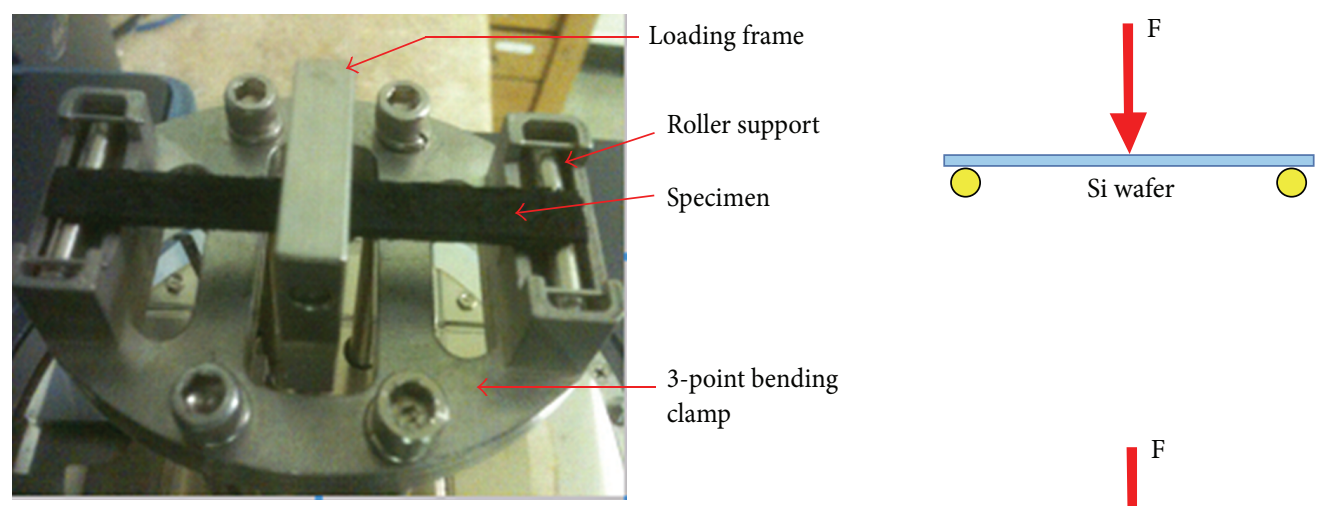

(a)
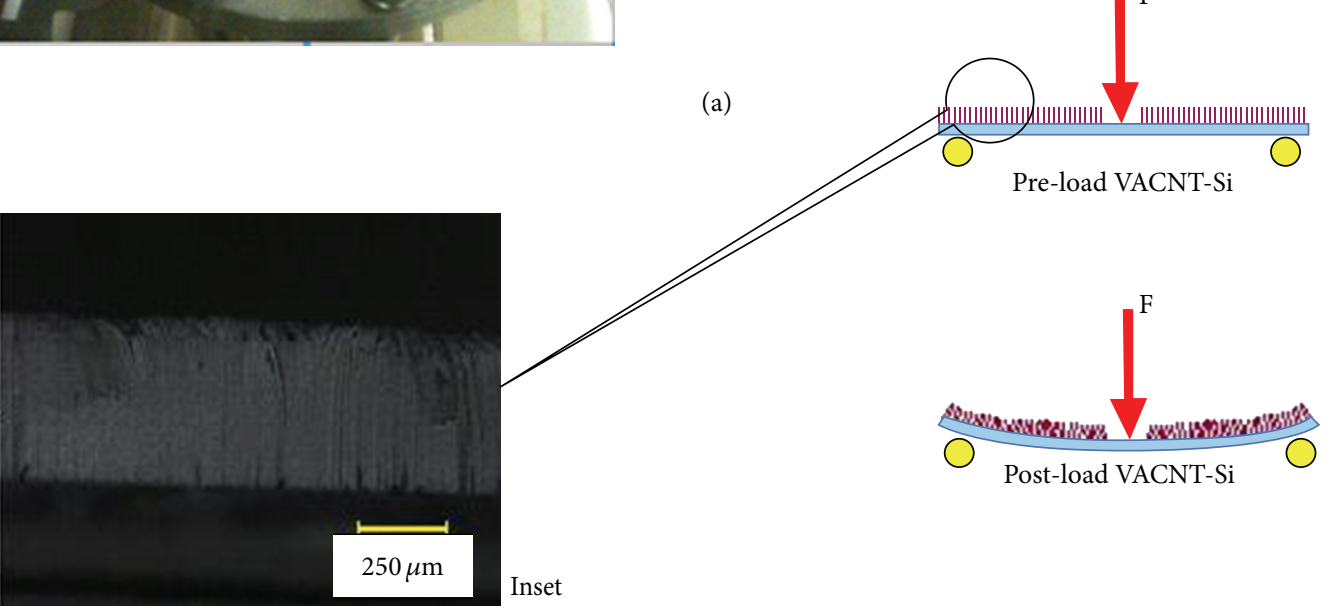

(b)

FIGURE 2: Experimental setup for DMA testing; (a) sample in three-point bending at a prespecified $10 \mu \mathrm{m}$ amplitude and $1 \mathrm{~Hz}$ oscillation frequency, and (b) energy dissipation caused by the entanglement of VACNTs on silicon wafer substrate during cyclic flexural deformation (inset: digital microscope image of VACNTs on silicon wafer substrate).

midspan of the beam. VACNT forest was carefully scraped off the specimen-roller contact areas, to avoid localized flexure of VACNTs under the line of centric loading and also over the two end lines of support (Figure 2(b)). All specimens were subjected to a temperature ramp from ambient $\left(30^{\circ} \mathrm{C}\right)$ to $120^{\circ} \mathrm{C}$, at a constant heating rate of $2^{\circ} \mathrm{C} \cdot \mathrm{min}^{-1}$ during the DMA testing. Raw data obtained from DMA experiments include the required amount of force $(F)$ and corresponding sample response-delay in phase angle $(\delta)$ for a prespecified $10 \mu \mathrm{m}$ amplitude at $1 \mathrm{~Hz}$ oscillation frequency. The dynamic mechanical parameters, such as storage modulus $\left(E^{\prime}\right)$, loss modulus $\left(E^{\prime \prime}\right)$, and $\tan \delta$ (damping loss factor, ratio of $E^{\prime \prime}$ to $\left.E^{\prime}\right)$, are estimated in data analysis.

The DMA stiffness model for a rectangular cross-section specimen under three-point bending load condition is given by [7]

$$
\begin{gathered}
E=K \cdot G, \\
K=\frac{F}{d}, \\
G=\left(\frac{L^{3}}{6 I}\right)\left[1+\frac{6}{10}(1+\nu)\left(\frac{t}{L}\right)^{2}\right], \\
E^{\prime}=E \cos \delta,
\end{gathered}
$$

where $E=$ elastic modulus, $K=$ estimated stiffness, $G=$ geometry factor, $F=$ measured force, $d=$ prespecified amplitude, $L=$ length (half span), $I=b t^{3} / 12$ (area moment of inertia of beam cross-section), $b=$ beam width, $t=$ thickness, and $v=$ specimen Poisson's ratio.

Whereas the required force $(F)$ and phase angle $(\delta)$ are true indicators of the test sample's flexural rigidity and damping the estimated storage modulus, however, is influenced by the data input for specimen thickness and Poisson's ratio. The pure silicon wafer, being anisotropic, has two different Poisson ratios, and there are no data available for the VACNTs. As such, a reported [8] in-plane Poisson's ratio of $\nu_{x y}=0.064$ for silicon has been used for both pure Si wafer and the VACNT-Si DMA test specimens. Regarding sample thickness, since the VACNT forest was scraped off the area where cyclic loading was applied, the effectual pure Si wafer thickness of $0.4 \mathrm{~mm}$ ( 0.016 inches) was also used as input data for the VACNT-Si specimen thickness. The ensuing "apparent" storage modulus from such approximations is provided later in the results section along with the required force $(F)$ and phase angle $(\delta)$, which are more accurate indicators of the test sample's flexural rigidity and damping.

2.3. High-Strain Rate Testing. The high-strain rate testing of pure Si wafer and VACNT-Si was conducted using 
a split-Hopkinson pressure bar (SHPB, also known as Kolsky Bar). It is capable of testing materials at high compressive strain rates typically ranging from $10^{2}$ to $10^{4}$ per second depending on the bar length and requirements of stress equilibrium and constant strain rate. The simplifying assumptions in the theory of SHPB are [9]

(a) bars are elastic,

(b) one-dimensional planar wave in the bars,

(c) uniaxial stress state in the specimen, and

(d) uniform state of stress in specimen, that is, negligible wave propagation effects.

The dynamic stress-strain, strain rate, and energy absorption can be obtained from the incident, reflected, and transmitted strain pulses. Based on force equilibrium considerations, the specimen strain $\left(\varepsilon_{s}\right)$ is computed from the reflected $\left(\varepsilon_{r}\right)$ and transmitted $\left(\varepsilon_{t}\right)$ pulses in

$$
\begin{gathered}
\varepsilon_{s}=\frac{C_{0}}{L} \int_{0}^{t}\left(\varepsilon_{t}-\varepsilon_{r}-\varepsilon_{r}-\varepsilon_{t}\right) d t \\
\varepsilon_{s}=\frac{-2 C_{0}}{L} \int_{0}^{t} \varepsilon_{r} d t
\end{gathered}
$$

where $C_{0}$ is the wave velocity in the Hopkinson bars and $L$ is the specimen length.

For a specimen of cross-sectional area $A_{s}$, the stress and the strain rate in the specimen are given by the following, respectively:

$$
\begin{aligned}
& \sigma_{s}=E \frac{A}{A_{s}} \varepsilon_{t}=K_{1} \varepsilon_{t}, \\
& \dot{\varepsilon}_{s}=\frac{-2 C_{0}}{L} \varepsilon_{r}=K_{2} \varepsilon_{r},
\end{aligned}
$$

where, $E$ and $A$ are Young's modulus and cross-sectional area of the Hopkinson bars, $K_{1}$ and $K_{2}$ are the stress and the strain rate multiplying factors for a given specimen and test setup. On superposition with the stress-versus-time data, the transient stress-strain response is obtained.

The energy absorbing capacity of the specimen is defined as the energy required for deforming a given specimen to a specific strain [10]. The absorption energy per unit volume up to ultimate failure strain $\varepsilon_{u}$ can be evaluated by integrating the area under the stress-strain curve for each specimen as shown in the following

$$
W=\int_{0}^{\varepsilon_{u}} \sigma_{s} d \varepsilon_{s} .
$$

The diameter of our SHPB is 0.75 inches $(19.05 \mathrm{~mm})$, so the maximum size of a square sample that can fit is 0.5 inches $(12.7 \mathrm{~mm})$ on each side. In the current study layered specimens that are a combination of soft and flexible (VACNTs) and very hard (silicon) materials are investigated. The soft VACNT forest layer considered by itself requires the use of low impedance bars made of materials such as aluminum or polycarbonate. However, due to the hard silicon wafer substrate, there is an increased possibility for the softer aluminum or polycarbonate bars to get scoured/dented, which necessitates the usage of harder maraging steel bars. There are no standards available in the literature for SHPB specimen design of this nature, and the challenge is to determine the optimal specimen dimensions by trial experiments.

Also the specimens need to be dimensioned such that the thickness-to-side ratio (aspect ratio) is optimized for a good response signal during SHPB testing. The aspect ratio of the specimen controls the strain rate as well. The range for aspect ratios is different for soft materials and hard materials $[9,11]$. For soft materials, the range of thickness/diameter aspect ratio (for circular specimens) is between 0.25 and 0.5 . In the case of metals it is between 0.5 and 1.0, and for ceramics it is between 1.0 and 2.0. There are other considerations as well that depend on the specimen dimensions such as stress equilibrium (thickness dependent), friction and radial inertia (diameter dependent).

The VACNT-Si specimens were placed with the Si substrate on the incident bar side and the VACNT coating facing the transmission bar side. Glycerin was applied as couplant between the Si substrate and the incident bar face. The transmission bar was carefully placed in contact with the VACNT forest layer surface without precompressing the forest layer. The tests were conducted at constant force (as determined by a reservoir pressure of 10 psi applied to the striker bar). Since this is a new material system, trials with various specimen sizes were evaluated. Specific results for square samples of approximate size $6 \mathrm{~mm}$ ( 0.24 inches $) \times 6 \mathrm{~mm}(0.24$ inches $)$ $\times 0.4 \mathrm{~mm}$ (0.016 inches) for the pure Si wafer and $6 \mathrm{~mm}(0.24$ inches $) \times 6 \mathrm{~mm}(0.24$ inches $) \times 0.93 \mathrm{~mm}$ ( 0.037 inches $)$ for VACNT-Si are reported here. Three specimens each of pure $\mathrm{Si}$ wafer and VACNT-Si processed at $820^{\circ} \mathrm{C}$ were tested in compression and the results analyzed.

\section{Results and Discussion}

Three specimens each of pure Si wafer and VACNT-Si were tested in the DMA under three-point oscillatory flexural load over a temperature range from ambient $\left(30^{\circ} \mathrm{C}\right)$ to $120^{\circ} \mathrm{C}$. The flexural rigidity and damping of these specimens were investigated in terms of the required force $(F$, line force at midspan to achieve $10 \mu \mathrm{m}$ amplitude, Figures 3(a) and $3(\mathrm{~b})$ ), "apparent" storage modulus $\left(E^{\prime}\right.$, Figures $3(\mathrm{c})$ and $3(\mathrm{~d}))$, and the damping loss factor $(\tan \delta$, ratio of dissipated energy to stored energy, Figures 3(e) and 3(f)). As can be observed, these values remain constant over the $120^{\circ} \mathrm{C}$ test temperature range, without a demonstrated peak of loss factor along with drop in storage modulus, which is more typical for viscoelastic materials around their glass transition temperature $[5,6]$.

Both the required force and "apparent" storage modulus show a negligible drop for the VACNT-Si specimens, but still within experimental scatter. This could perhaps be attributed to some degradation and/or chemical erosion of specimen surface during the growth of VACNTs at an elevated $820^{\circ} \mathrm{C}$. On the other hand, damping loss factor $(\tan \delta)$ 


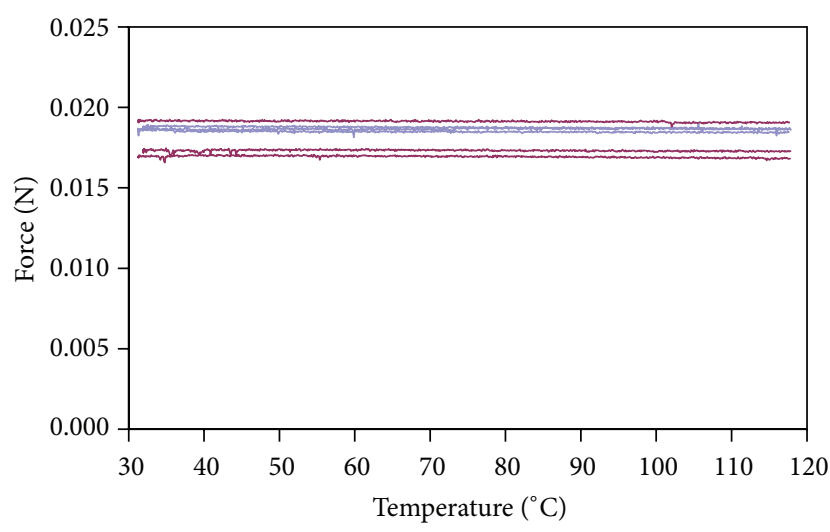

(a)

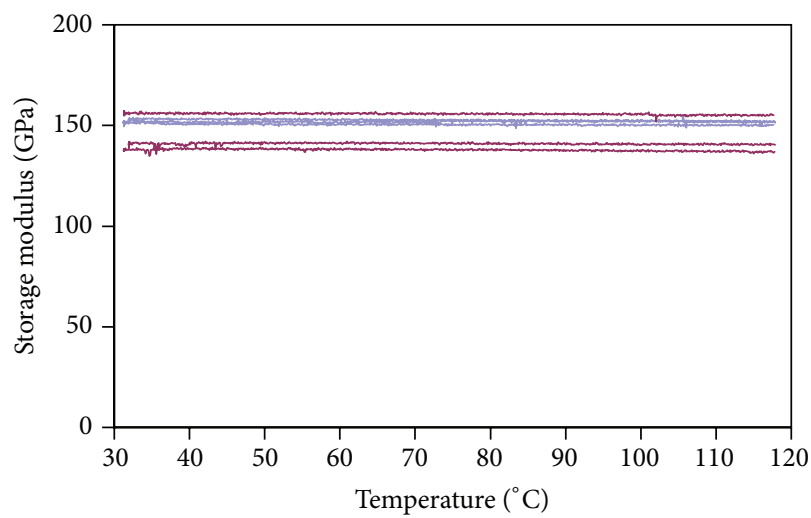

(c)

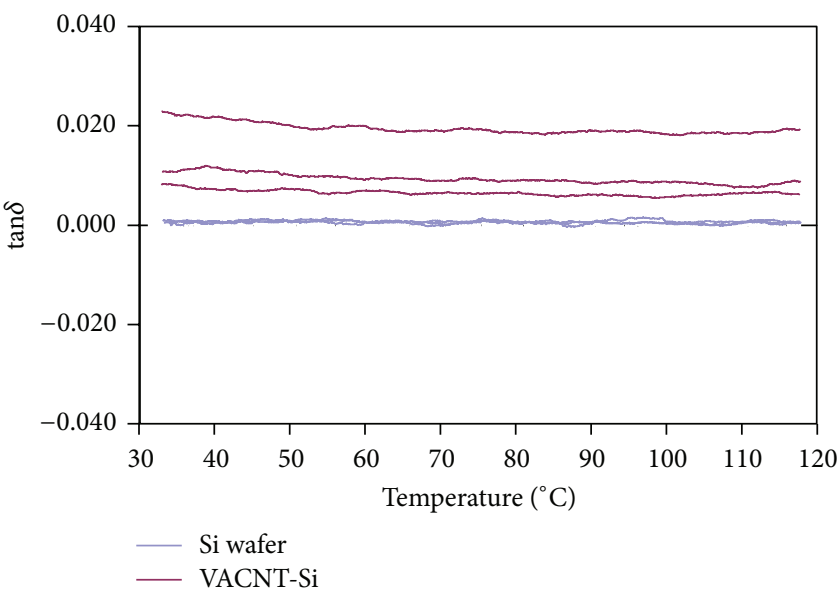

(e)

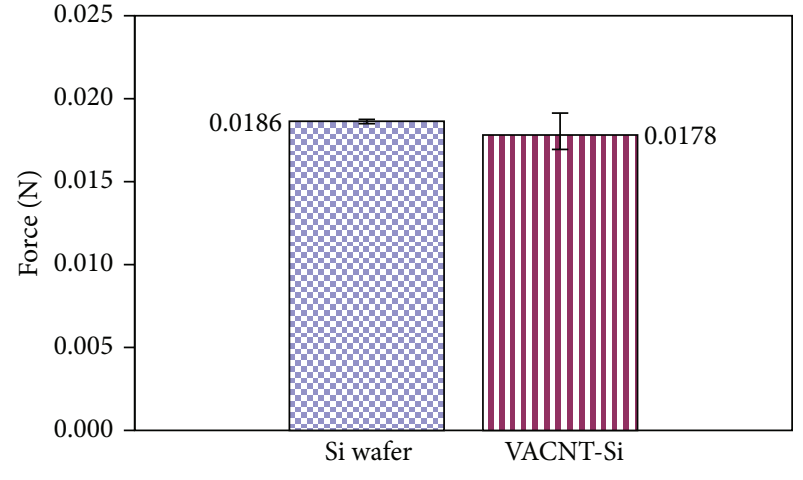

(b)

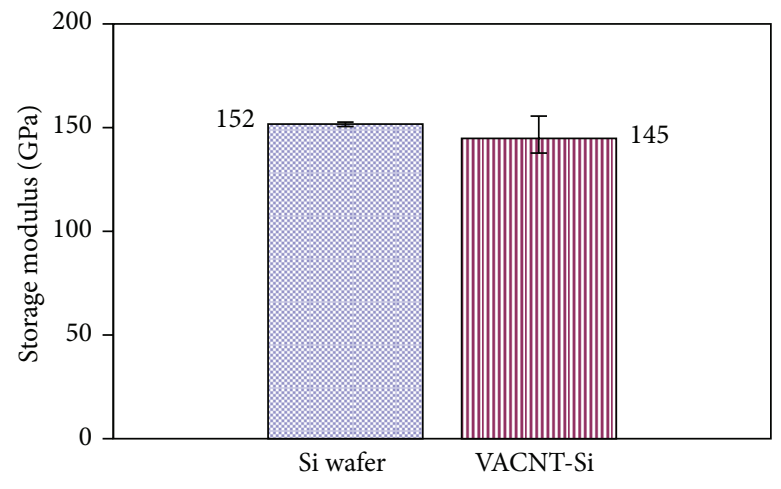

(d)

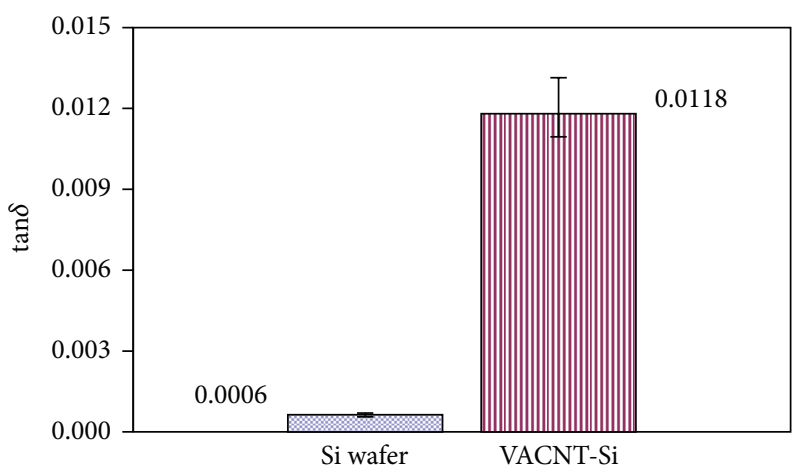

Figure 3: DMA results for three specimens each of pure Si wafer and VACNT-Si (processed at $820^{\circ} \mathrm{C}$ ) analyzed over a temperature range from ambient $\left(30^{\circ} \mathrm{C}\right)$ to $120^{\circ} \mathrm{C}$; ((a) and (b)) the required line force $F$ at midspan to achieve $10 \mu \mathrm{m}$ amplitude; ((c) and (d)) the "apparent" storage modulus, $E^{\prime}$; and ((e) and (f)) associated damping loss factor, tan $\delta$.

shows a remarkable increase of about $1800 \%$ for the samples with VACNTs grown on pure Si wafer (Figures 3(e) and 3(f)). Interfacial friction between individual VACNTs (with very large surface area to diameter), caused by their entanglements under cyclic deformation (Figure 2(b)), is believed to be the primary energy dissipation mechanism for such high improvement in loss factor.
As mentioned previously, the combination of the soft VACNT forest layer with the hard silicon wafer presented novel challenges for SHPB testing. The VACNT forest layer is typical of an open-cell foam structure consisting of well-arranged one-dimensional units [12]. Various energy absorption mechanisms such as localized higher mode buckling of VACNTs under static compressive loading and 


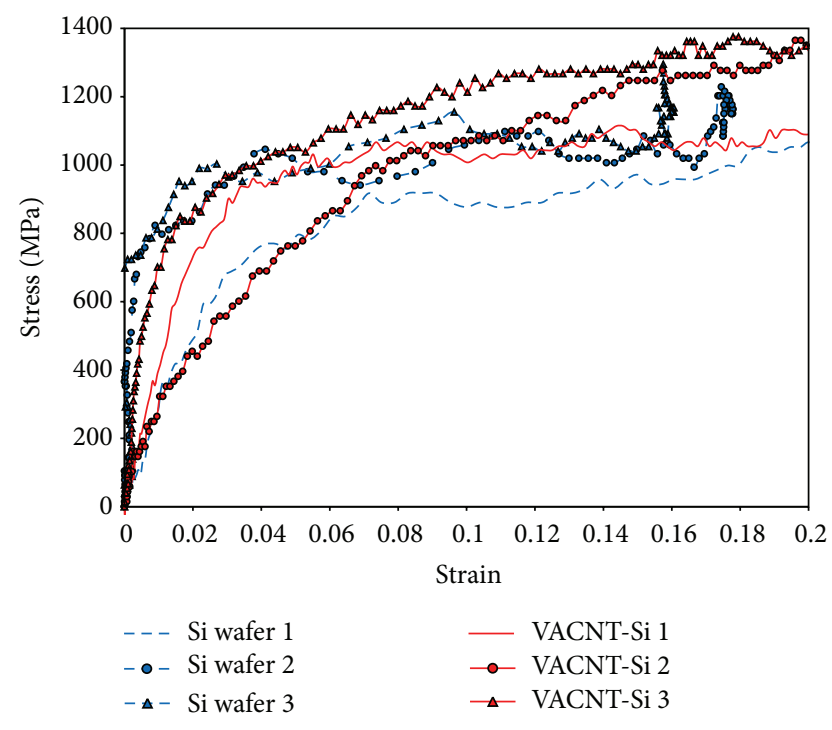

Figure 4: High strain rate response of pure Si wafer and VACNT-Si (processed at $820^{\circ} \mathrm{C}$ ) over the strain rate range of $4600 / \mathrm{s}$ to $7500 / \mathrm{s}$ (shown only up to 0.2 strain).

nanoindentation along with surface entanglement and van der Waals interactions with neighboring nanotubes have been reported in the literature $[13,14]$. To the authors' knowledge, this type of layered material combination with such a contrast in properties has not been tested using SHPB for analyzing the high-strain rate dynamic response.

It was observed that the pure $\mathrm{Si}$ wafer specimens achieve a lower stress for a given strain, as compared to VACNTSi specimens for average strain rates ranging from 4600/s to $7500 / \mathrm{s}$ (Figure 4 ). Since the nanoreinforced functionally graded material systems are being developed for light-weight structural applications, the specific energy absorption given by the ratio of energy absorbing capacity (5) to the mass density is reported in Figures 5 and 6.

An average increase of around $250 \%$ in specific energy absorption (Joules/Kg) was observed for VACNT-Si layered specimens in comparison with pure $\mathrm{Si}$ wafer specimens for the strain rates mentioned above (Figure 5). It was also observed that the VACNT-Si specimens achieved an average increase of around $450 \%$ in the rate of specific energy absorption (Joules/ $\mathrm{Kg} / \mathrm{sec}$ ), when compared with pure Si wafer (Figure 6).

\section{Conclusion}

The dynamic mechanical behavior and high-strain rate dynamic response characteristics of a functionally graded material system consisting of vertically aligned carbon nanotube ensembles grown on a silicon wafer substrate (VACNT$\mathrm{Si})$ have been investigated. The functionally graded VACNTSi exhibited significantly higher damping without sacrificing the flexural rigidity. Interfacial friction between individual VACNTs caused by their entanglements under cyclic deformation is believed to be the primary energy dissipation mechanism for such a large improvement in loss factor.

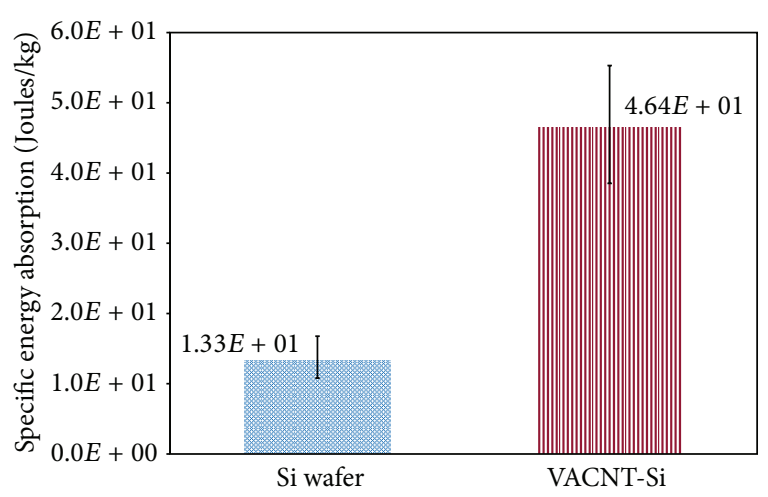

FIgURE 5: Specific energy absorption of pure Si wafer and VACNT-Si (processed at $820^{\circ} \mathrm{C}$ ).

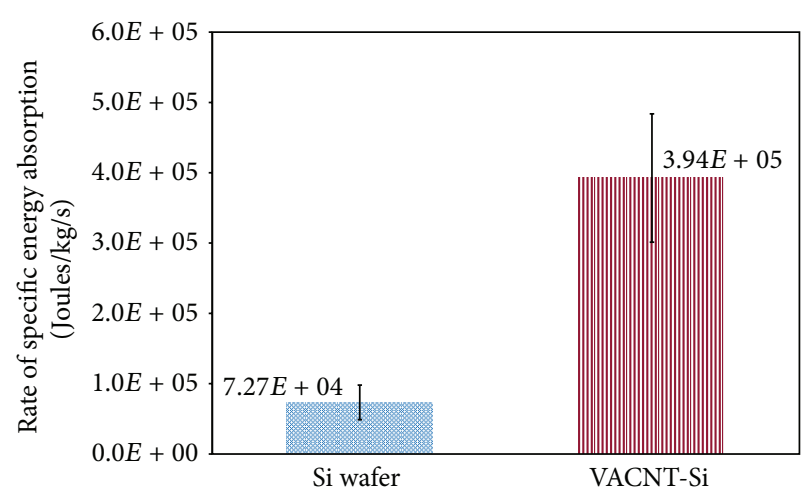

FIGURE 6: Rate of specific energy absorption for pure Si wafer and VACNT-Si (processed at $820^{\circ} \mathrm{C}$ ).

In the case of high-strain rate compressive loading, a large increase in specific energy absorption as well as the rate of specific energy absorption was observed for VACNT-Si layered specimens as compared with pure Si wafer.

\section{Acknowledgment}

This research was funded by Grant no. W9132T-12-P0057 from the US Army Engineer Research and Development Center-Construction Engineering Research Laboratory (ERDC-CERL), Champaign, IL, USA. The authors would like to thank Mr. Damian Stoddard and Mr. Kiyun Kim, mechanical engineering graduate students at the University of Mississippi, for their help with the DMA and Hopkinson bar experiments.

\section{References}

[1] C. M. Seah, S. P. Chai, and A. R. Mohamed, "Synthesis of aligned carbon nanotubes," Carbon, vol. 49, no. 14, pp. 4613-4635, 2011.

[2] Y. J. Jung, B. Q. Wei, R. Vajtai et al., "Mechanism of selective growth of carbon nanotubes on $\mathrm{SiO}_{2} / \mathrm{Si}$ patterns," Nano Letters, vol. 3, no. 4, pp. 561-564, 2003.

[3] C. Daraio, V. F. Nesterenko, and S. Jin, "Highly nonlinear contact interaction and dynamic energy dissipation by forest of 
carbon nanotubes," Applied Physics Letters, vol. 85, no. 23, pp. 5724-5726, 2004.

[4] S. Handuja, S. P. Singh, P. Srivastava, and V. D. Vankar, "Growth of long aligned carbon nanotubes on amorphous hydrogenated silicon nitride by thermal chemical vapor deposition," Materials Letters, vol. 63, no. 15, pp. 1249-1251, 2009.

[5] Y. Zeng, L. Ci, B. J. Carey, R. Vajtai, and P. M. Ajayan, "Design and reinforcement: vertically aligned carbon nanotube-based sandwich composites," ACS Nano, vol. 4, no. 11, pp. 6798-6804, 2010.

[6] B. Pramanik and P. R. Mantena, "Viscoelastic response of graphite platelet and CTBN reinforced vinyl ester nanocomposites," Materials Sciences and Applications, vol. 2, no. 11, pp. 16671674, 2011.

[7] Dynamic Mechanical Analyzer, Q Series, Calculation Based on Clamp Type, Revision F, TA Instruments, New Castle, Del, USA, 2004.

[8] M. A. Hopcroft, W. D. Nix, and T. W. Kenny, "What is the Young's modulus of silicon?" Journal of Microelectromechanical Systems, vol. 19, no. 2, Article ID 5430873, pp. 229-238, 2010.

[9] B. A. Gama, S. L. Lopatnikov, and J. W. Gillespie Jr., "Hopkinson bar experimental technique: a critical review," Applied Mechanics Reviews, vol. 57, no. 4, pp. 223-250, 2004.

[10] F. Yi, Z. Zhu, F. Zu, S. Hu, and P. Yi, "Strain rate effects on the compressive property and the energy-absorbing capacity of aluminum alloy foams," Materials Characterization, vol. 47, no. 5, pp. 417-422, 2001.

[11] B. Song and W. Chen, "Split Hopkinson pressure bar techniques for characterizing soft materials," Latin American Journal of Solids and Structures, vol. 2, no. 2, pp. 113-152, 2005.

[12] A. Cao, P. L. Dickrell, W. G. Sawyer, M. N. Ghasemi-Nejhad, and P. M. Ajayan, "Super-compressible foamlike carbon nanotube films," Science, vol. 310, no. 5752, pp. 1307-1310, 2005.

[13] T. Tong, Y. Zhao, L. Delzeit, M. A. Kashani, M. Meyyappan, and A. Majumdar, "Height independent compressive modulus of vertically aligned carbon nanotube arrays," Nano Letters, vol. 8, no. 2, pp. 511-515, 2008.

[14] S. B. Hutchens, A. Needleman, and J. R. Greer, "Analysis of uniaxial compression of vertically aligned carbon nanotubes," Journal of the Mechanics and Physics of Solids, vol. 59, no. 10, pp. 2227-2237, 2011. 

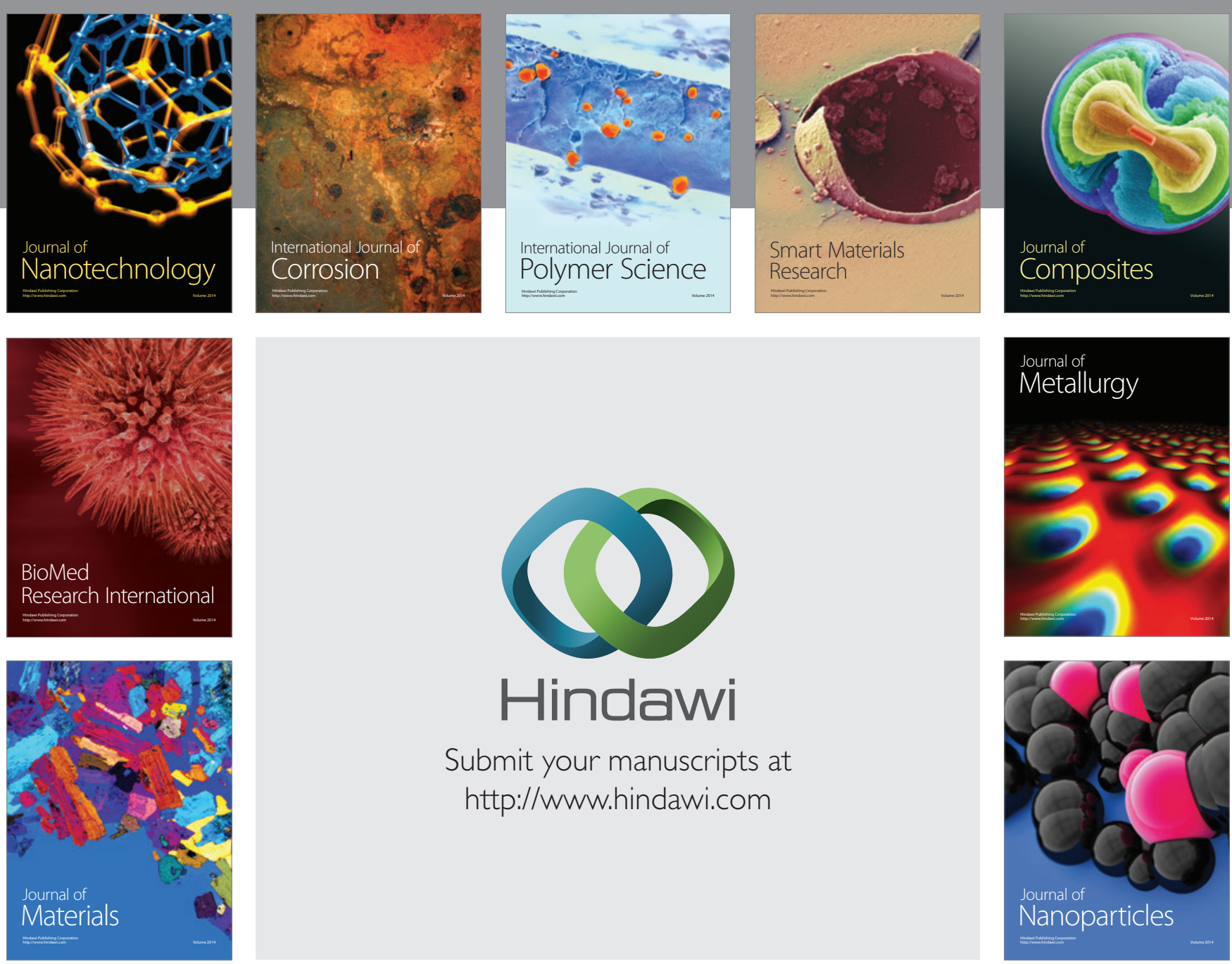

Submit your manuscripts at http://www.hindawi.com
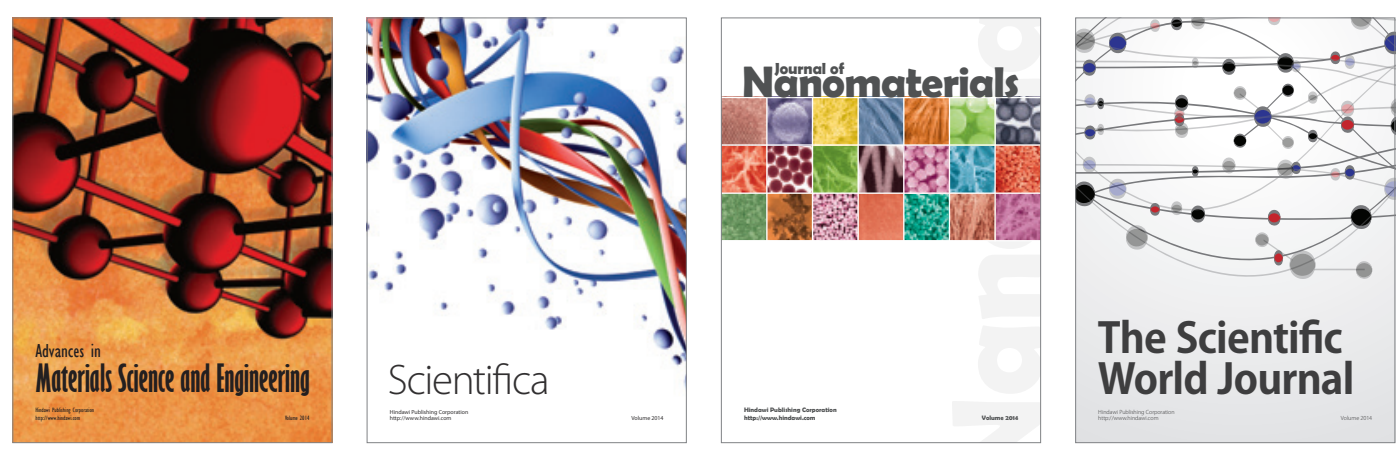

\section{The Scientific World Journal}
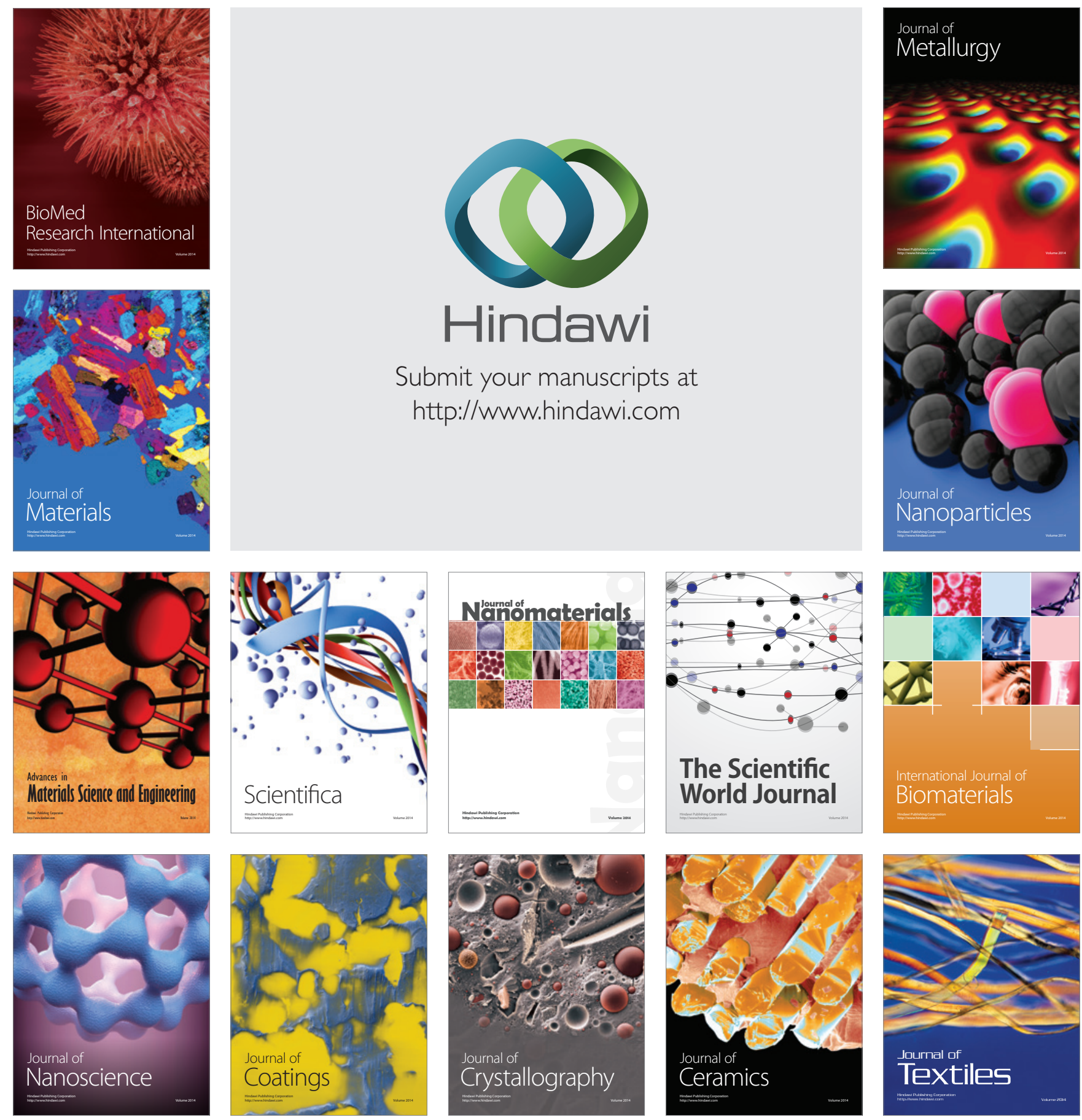